\title{
Resonant Magnetic X-ray Scattering from Terbium
}

\author{
J. Strempfer ${ }^{1}$, D. Hupfeld ${ }^{2}$, J. Voigt ${ }^{2}$, G. Bihlmayer ${ }^{2}$, A.I. \\ Goldman $^{3}$, and Th. Brückel ${ }^{2}$ \\ ${ }^{1}$ Hamburger Synchrotronstrahlungslabor HASYLAB at Deutsches \\ Elektronensynchrotron DESY, 22503 Hamburg, Germany \\ ${ }^{2}$ Institut für Festkörperforschung at Forschungszentrum Jülich, 52425 Jülich, \\ Germany \\ ${ }^{3}$ Ames Laboratory, USDOE and Department of Physics and Astronomy, Iowa State \\ University, Ames, IA 50011, USA \\ E-mail: joerg.strempfer@desy.de
}

\begin{abstract}
Resonant magnetic $\mathrm{x}$-ray scattering from $\mathrm{Tb}$ in the spiral phase is studied in great detail. Polarization analysis in the $\sigma-\sigma^{\prime}$ and $\sigma-\pi^{\prime}$ channels has been performed for magnetic $(0,0, l \pm \tau)$ satellite reflections over the accessible wave vector range $\mathrm{Q}=1.9-6.5 \AA^{-1}$. A characteristic splitting of the resonance signal as a function of energy has been observed at the $\mathrm{L}_{\mathrm{II}}$ as well as at the $\mathrm{L}_{\mathrm{III}}$ absorption edge. The shape of the resonance depends on $\mathrm{Q}$ and is different for the two edges. Up to 3 components were observed with a separation in energy between 3.3 and $4.9 \mathrm{eV}$, compared to a core hole lifetime broadening of $2.5 \mathrm{eV}$, which are in agreement with the density of states in Tb-metal.
\end{abstract}

PACS numbers: 78.70.Ck, 75.50.-y, 75.50.Ec

Submitted to: J. Phys.: Condens. Matter 


\section{Introduction}

The first resonant magnetic x-ray scattering experiment from an antiferromagnet was performed on Ho $[1,2]$ and revealed new features not found in previous neutron scattering experiments, such as spin-slip discommensurations in the helical phase. This study initiated a re-investigation of the magnetic structure of the rare-earth elements $[3,4,5,6,7,8,9,10]$. The available data now provide a wealth of information about the resonance scattering process itself. Hannon et al. interpreted the scattering process in an atomic-like model [11]. In second order perturbation theory for the coupled system of magnetic atom and the electromagnetic wave field, the observed resonances can be described as virtual transitions induced by the incident photons from core states to excited states with subsequent coherent reemission. According to [11], the process becomes sensitive to the magnetic state due to the exchange splitting of the intermediate excited states, hence the name "X-ray Resonance Exchange Scattering" (XRES). To emphasize the relation to magnetism, we will use here the less specific term "Resonant Magnetic X-ray Scattering" (RMXS). The extent to which solid state properties must be taken into account is still an open issue. Compared to a simple model description in terms of atomic-like wave functions, a number of factors can influence the strength and resonance line shape of RMXS in the solid, such as the electronic band structure (density of states and modified radial wave functions), the hybridization and exchange interaction between bands, the spin-orbit coupling, and crystal-electric field effects. In the present study, we present resonant scattering data which clearly reflect features of the band structure of the electronic states for terbium metal. The situation for the lanthanides can be summarized as follows: strong resonance enhancements are observed at the $\mathrm{L}_{\mathrm{II}}$ and $\mathrm{L}_{\mathrm{III}}$ edges, while the enhancement at the $\mathrm{L}_{\mathrm{I}}$ edge is negligible due to the small overlap between the $1 s$ core states and the relevant intermediate states. The so- called "branching ratio", i. e. the ratio between the intensity enhancement at the $\mathrm{L}_{\mathrm{III}}$ and $\mathrm{L}_{\mathrm{II}}$ edges, varies continuously throughout the lanthanide series [12], with values larger than one for the light rare earths, approximately one for the S-state of Gd [13] and smaller than one for the heavy rare earths. Recently, the branching ratio for the heavy rare earths has been explained in terms of the role of spin-orbit coupling in the 5d-band [14]. Polarization analysis shows that the dominant transitions are of dipolar character. In the atomic model they consist of the $2 p \rightarrow 5 d$ transition. A spin polarization of the $5 d$ band induced by the magnetic $4 f$ levels is required for a sensitivity to the magnetic state of the atom. Within the limits given by the lifetime broadening of the intermediate excited states of about $2-4 \mathrm{eV}$, fine structures of the electronic levels were not observed in most cases and the resonance-line shape could well be approximated by a model assuming transitions between two sharp atomic-like electronic levels. One notable exception is metallic $\mathrm{Tb}$ [4]. In addition to the dipolar transitions, quadrupolar transitions $2 p \rightarrow 4 f$ shifted with respect to the dipolar resonances to smaller energies are possible. In general, these quadrupole resonances are weaker than the dipolar resonances and are not observed for all rare earth metals. 
As we will show in this paper, magnetic resonant scattering from terbium in its antiferromagnetic state does not fit into this general picture. The experiments on $\mathrm{Tb}$ are rather challenging, since the magnetic moment in the antiferromagnetic, helical state below $\mathrm{T}_{\mathrm{N}} \sim 230 \mathrm{~K}$ remains very small before a transition into a basal plane ferromagnetic state occurs at $\mathrm{T}_{\mathrm{C}} \sim 220 \mathrm{~K}$. In the first resonance experiments on $\mathrm{Tb}$ $[15,16]$, only the stronger $\mathrm{L}_{\mathrm{III}}$ resonance was observed which seemed to behave normally. In a subsequent study [4], the resonance at the $\mathrm{L}_{\mathrm{II}}$ edge was also investigated. The observations indicated a splitting of the $\mathrm{L}_{\mathrm{II}}$-resonance in energy. The authors modeled the Q-dependence of the splitting in terms of one quadrupolar and one dipolar transition and attempted a polarization analysis at one Q-position. Despite these efforts, the origin of the observed double resonance remains controversial. So far no experiment has been conducted to investigate the resonant scattering in the $\sigma-\sigma^{\prime}$ and $\sigma-\pi^{\prime}$ channel separately at different positions in reciprocal space. Without polarisation analysis it is not possible to determine the nature of the scattered intensities in the different peaks, e.g. dipolar or quadrupolar, the first step to determine the origin of the scattering observed. In what follows, we will present results of such a complete polarization study. These data have been obtained on a third generation synchrotron source where the energy resolution compared to the second generation source was improved by a factor 3. This is of great importance in the case of the double resonance in terbium since the peak splitting is only about $4.5 \mathrm{eV}$.

\section{Experimental}

The RMXS experiments presented here were conducted at the undulator beamlines 12ID-B (BESSRC-CAT) and 6-ID-B (MU-CAT) at the Advanced Photon Source (APS) at Argonne National Laboratory. Preliminary studies with polarization analysis were conducted at the wiggler beamline W1 at HASYLAB/DESY. Here, the incident beam was approximately $94 \%$ linearly polarized and had an energy spread of about $3.3 \mathrm{eV}$. In the experiments conducted at the APS, the incident beam was $99.9 \%$ linearly polarised perpendicular to the vertical diffraction plane and the energy spread was as low as $1.3 \mathrm{eV}$. The photon energy was tuned to the terbium $\mathrm{L}_{\mathrm{II}}(8252 \mathrm{eV})$ and $\mathrm{L}_{\mathrm{III}}$ edges $(7514$ $\mathrm{eV})$.

The terbium crystal we used is a cube with dimensions of $4 \times 4 \times 4 \mathrm{~mm}^{2}$. Because of the relatively low penetration depth of $3 \mu \mathrm{m}$ at the $\mathrm{L}_{\mathrm{II}}$ edge, the $\left(\begin{array}{llll}0 & 0 & 0 & 1\end{array}\right)$-face (in what follows, we use the Miller indices $h k l$ ) was etched with $\mathrm{HNO}_{3}$ to remove the oxide layer just before the crystal was mounted in a closed-cycle cryostat. The rocking curve width of the crystal after etching was $0.18^{\circ}$.

Polarization analysis in the $\sigma-\pi^{\prime}$ channel was performed using a pyrolytic graphite crystal. The ( $\left.\begin{array}{lll}0 & 0 & 6\end{array}\right)$-reflection of the PG-analyzer crystal $(2 d=2.236 \AA)$ gives a diffraction angle of $2 \theta=84.4^{\circ}$ and $2 \theta=95.1^{\circ}$ for the $\mathrm{L}_{\mathrm{II}}$ and $\mathrm{L}_{\mathrm{III}}$ edge, respectively. This results in a suppression of the $\sigma-\sigma^{\prime}$ scattering by two orders of magnitude. A comparison between the measured resonance line shapes taken from the same crystal in 
the same configuration at DORIS/HASYLAB (second generation synchrotron source) and APS (third generation synchrotron source) is shown in figure 1, where the absorption corrected intensity of the (0 $04+\tau)$ magnetic superstructure peak is shown (in both cases measured in the $\sigma-\pi^{\prime}$ channel). The energy resolution in the two experiments differs by about a factor of 3 . The figure clearly demonstrates the superiority of a modern source: the splitting, which is only hinted in the DORIS III data is very pronounced in the APS data and the statistics is significantly improved.

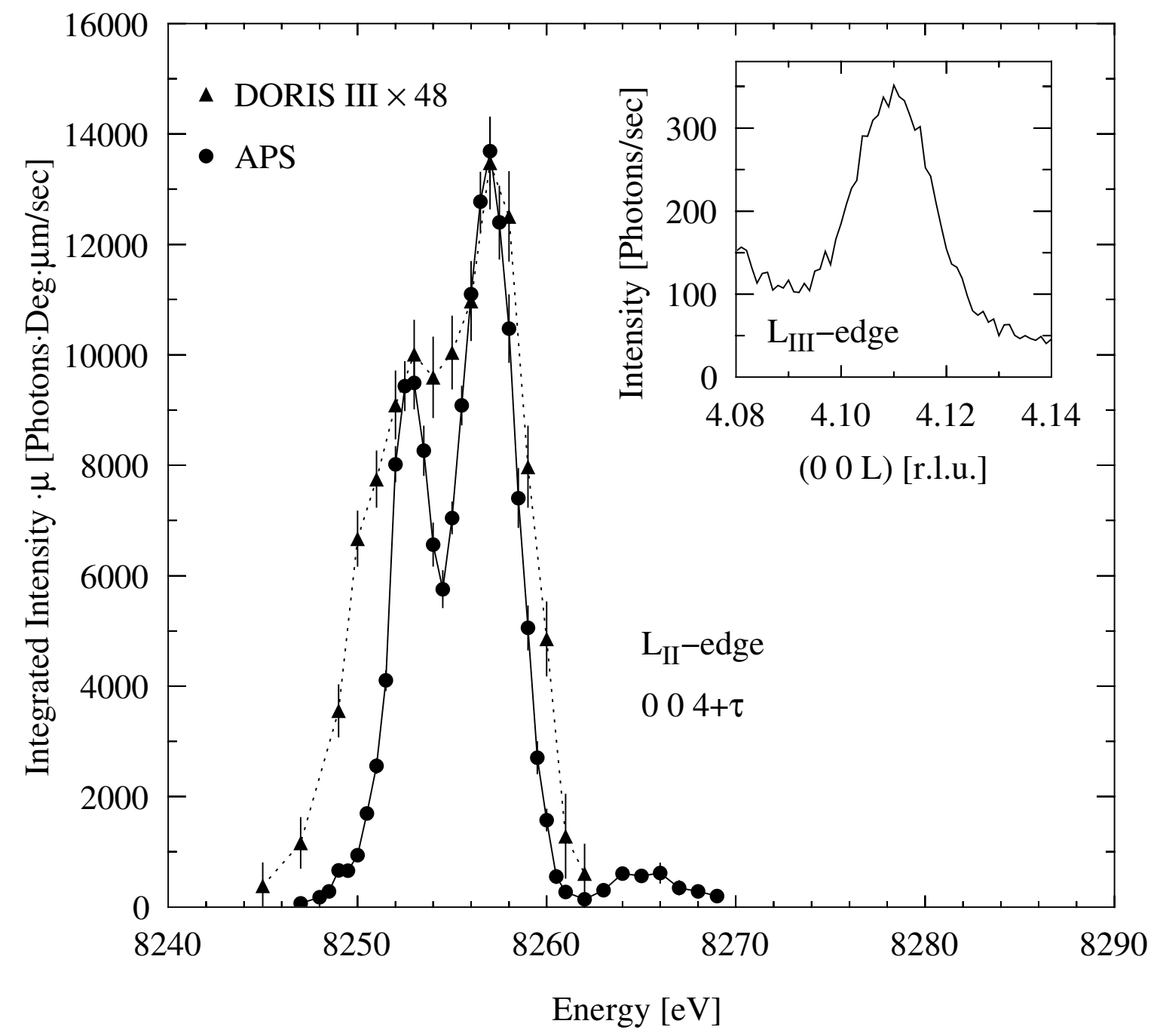

Figure 1. Comparison of the $(0,0,4+\tau)$ magnetic reflection of $\mathrm{Tb}$ at a second $(\mathbf{\Lambda})$ and a third generation synchrotron source $(\bullet)$ measured with $\sigma-\pi^{\prime}$ polarization analysis after correction for absorption. The DORIS III data have been multiplied by a factor of 48 to be scaled to the APS data. The solid and dashed lines are guides to the eye. The insert shows raw data from an l-scan over the magnetic $(0,0,4+\tau)$ satellite at the peak maximum of the $\mathrm{L}_{\mathrm{III}}$ resonance at $7.482 \mathrm{keV}$ at a sample temperature of $T=224 \mathrm{~K}$, measured at the APS.

Terbium crystallizes in a hexagonal close-packed structure with space group $P 3 / m m c$ and RT lattice-parameters of $a=3.599 \AA$ and $c=5.696 \AA$ [17]. It undergoes 
a phase transition from the paramagnetic to an antiferromagnetic state at $\mathrm{T}_{\mathrm{N}}=230 \mathrm{~K}$ and another phase transition to a ferromagnetic phase at $\mathrm{T}_{\mathrm{C}}=220 \mathrm{~K}$. In the antiferromagnetic phase the magnetic moment is ordered in a basal-plane helix. The turn angle varies with temperature between $16.7^{\circ}$ and $21.3^{\circ}$ from plane to plane resulting in a propagation vector from $\tau=0.0925(\mathrm{~T}=221 \mathrm{~K})$ to $\tau=0.1183(\mathrm{~T}=230 \mathrm{~K})$. The modulation wave vector is directed along $\left(\begin{array}{lll}0 & 0 & l\end{array}\right)$. Thus, the experiment was focused on the investigation of satellites of type $\left(\begin{array}{lll}0 & 0 & l\end{array} \tau\right)$.

The basis vectors of the polarization for the incident and the diffracted beam with respect to the crystal axis are defined as shown in figure 2. In our geometry, the magnetic moment lies within the $\hat{U}_{1}-\hat{U}_{2}$-plane with the propagation vector along $\hat{U}_{3}$.

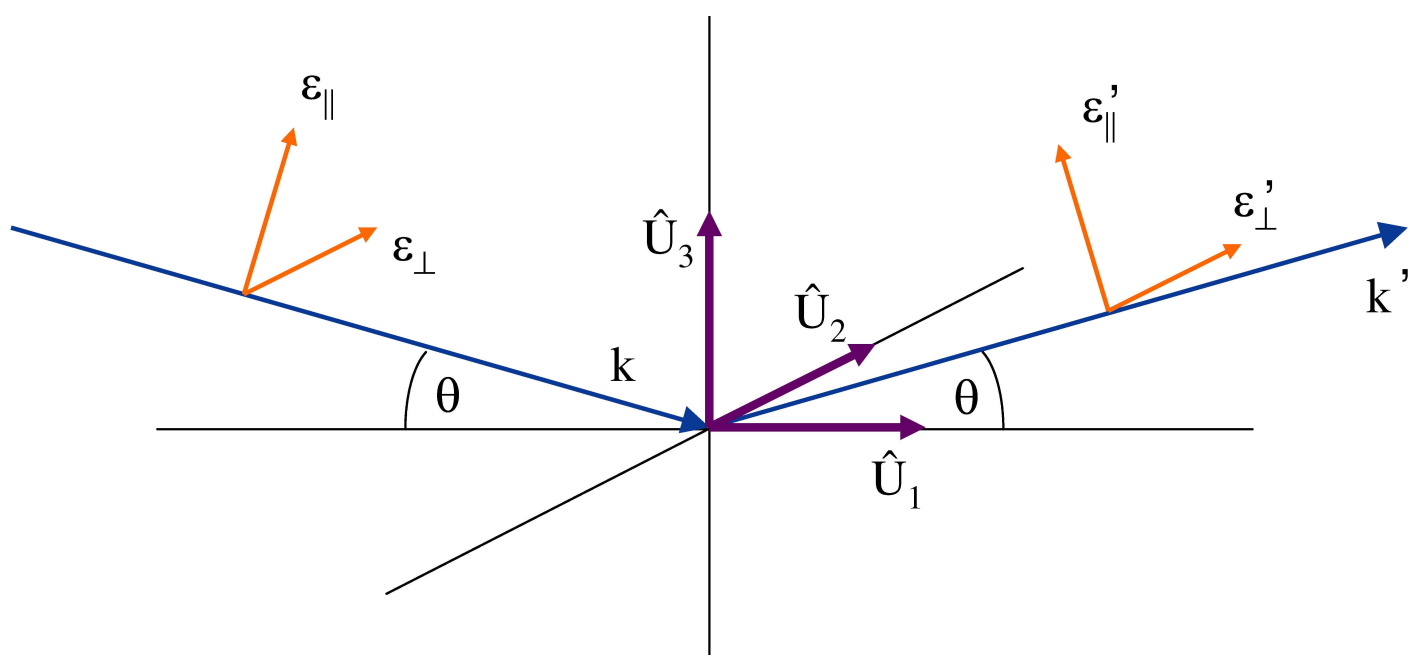

Figure 2. Scattering geometry and polarization with respect to the crystal geometry. $\hat{U}_{1}, \hat{U}_{2}$ and $\hat{U}_{3}$ denote the basis vectors of the magnetic structure and $\varepsilon_{\perp / \|}\left(\varepsilon_{\perp / \|}^{\prime}\right)$ the incoming (scattered) polarization perpendicular $(\sigma)$ and parallel $(\pi)$ to the diffraction plane.

According to [18] we can write the cross-section for ( $00 l \pm \tau)$ reflections for purely dipolar scattering from a basal-plane helix and perfectly linearly polarized incident radiation perpendicular to the scattering plane as:

$$
\begin{aligned}
d \sigma /\left.d \Omega\right|_{E 1} ^{r e s} & =\left(F^{(2)}\right)^{2} \delta(Q-G) \\
& +\frac{1}{4} \cos ^{2} \theta\left(F^{(1)}\right)^{2} \delta(Q-G \pm \tau) \\
& +\frac{1}{16}\left(1+\sin ^{2} \theta\right)\left(F^{(2)}\right)^{2} \delta(Q-G \pm 2 \tau)
\end{aligned}
$$

where $F^{(1)}$ and $F^{(2)}$ describe the transition probabilities from initial to intermediate states for the first and second order reflections, respectively, determining the strength of the resonance. We want to emphasize that these transition probabilities are independent of the momentum transfer, but depend strongly on the properties of the intermediate states as for example on the inverse lifetime $\Gamma$. The studies presented here are limited to the first order satellites which are described by the second term in (1). For our study 
of the resonance process itself, the quantity of interest is the strength $F^{(1)}$ of a given resonance. Apart from an insignificant constant (scaling factor) $F^{(1)}$ can be determined from the integrated intensity $I_{\text {meas }}=I_{\text {meas }}(E, G \pm \tau)$ of a satellite reflection $G \pm \tau$ according to

$$
\left|F^{1}\right|^{2}=I_{\text {meas }} T^{-1} L^{-1} /\left(T_{D W} \cos ^{2} \theta\right)
$$

with $T$ the transmission, $L^{-1}=\sin (2 \theta)$ the inverse Lorentz-factor, $T_{D W}$ the DebyeWaller factor and the cosine term in (1), accounting for the spin direction with respect to the polarization. The variation of the absorption coefficient $\mu$ over the $\mathrm{L}_{\mathrm{II}}$ and $\mathrm{L}_{\mathrm{III}}$ edges has been calculated from the fluorescence of the Tb-sample and a Tb-foil according to the formalism described in [13]. The necessary coefficients $\mu_{1}, \mu_{2}$ and $\mu_{F}$ have been calculated with the program package absorption by Brennan and Cowan [19]. The absorption corrections have then been applied to the data. In our case, where the incoming and scattered beam are symmetric to the crystal surface, the measured intensities have simply to be multiplied by the energy dependence of $\mu$ over the edge to obtain the corrected intensities [13].

A temperature dependence of the magnetic intensity has been measured and the maximum intensity was found at $224 \mathrm{~K}$. The measurements presented in the following have been conducted at this temperature, where we found $\tau=0.11$ (see insert figure 1). The behaviour of the magnetic intensity with temperature found here supports the results reported earlier $[20,4]$.

At the $\mathrm{L}_{\mathrm{III}}$ edge, energy scans have been performed to measure the energy dependence of the magnetic intensities. At some positions, a huge energy dependent background was found. Therefore, the same energy scan has been performed at $224 \mathrm{~K}$, in the antiferromagnetic phase, and at $231 \mathrm{~K}$ in the paramagnetic phase, where the magnetic signal has vanished. The purely magnetic signal was obtained by a subtraction of the two scans. At the $\mathrm{L}_{\mathrm{II}}$ edge, at each energy point a complete rocking curve has been taken. To make sure that both approaches lead to the same result, rocking curve scans at some energies were performed as well at the $\mathrm{L}_{\mathrm{III}}$ edge. The comparison of the data obtained with the two different methods showed that subtracting the background measured above the phase transition to obtain the magnetic signal is completely justified.

\section{Description of resonant magnetic x-ray scattering}

The resonance peak shape for RMXS is described in [13]. The scattering cross section is proportional to the square of the sum of the scattering amplitudes for charge scattering $\left(A_{C}\right)$, non-resonant magnetic scattering $\left(A_{M}\right)$ and resonant magnetic scattering $\left(A_{R}\right)$ :

$$
\frac{d \sigma}{d \Omega} \sim\left|A_{C}+i\left(A_{M}+\frac{A_{R}}{E} \frac{1}{\left(E-E_{0}\right)-i \frac{\Gamma}{2}}\right)\right|^{2}
$$

where $E_{0}$ denotes the edge energy and $\Gamma$ the inverse lifetime of the intermediate state. Since in terbium we deal with two peaks in energy, the sum of the scattering amplitudes 
for both peaks has to be introduced. For the description of the magnetic superlattice peaks, the charge term can be neglected since there is no charge signal at these positions. The resonant magnetic scattering amplitudes and the non-resonant scattering amplitude are assumed to be real and are added giving rise to an interference term between the resonance terms:

$$
\begin{aligned}
\frac{d \sigma}{d \Omega} & \sim\left|A_{M}+\frac{A_{1}}{E} \frac{1}{\left(E-E_{1}\right)-i \frac{\Gamma_{1}}{2}}+\frac{A_{2}}{E} \frac{1}{\left(E-E_{2}\right)-i \frac{\Gamma_{2}}{2}}\right|^{2} \\
& \sim A_{M}^{2} \\
& +\left(\frac{A_{1}}{E}\right)^{2} \frac{1}{\left(E-E_{1}\right)^{2}+\left(\frac{\Gamma_{1}}{2}\right)^{2}} \\
& +\left(\frac{A_{2}}{E}\right)^{2} \frac{1}{\left(E-E_{2}\right)^{2}+\left(\frac{\Gamma_{2}}{2}\right)^{2}} \\
& +\frac{2 A_{M}}{E}\left(\frac{A_{1}\left(E-E_{1}\right)}{\left(E-E_{1}\right)^{2}+\left(\frac{\Gamma_{1}}{2}\right)^{2}}+\frac{A_{2}\left(E-E_{2}\right)}{\left(E-E_{2}\right)^{2}+\left(\frac{\Gamma_{2}}{2}\right)^{2}}\right) \\
& +\left|\frac{A_{1} A_{2}}{E^{2}}\right| \frac{\left(E-E_{1}\right)\left(E-E_{2}\right)+\frac{\Gamma_{1} \Gamma_{2}}{4}}{\left[\left(E-E_{1}\right)^{2}+\left(\frac{\Gamma_{1}}{2}\right)^{2}\right]\left[\left(E-E_{2}\right)^{2}+\left(\frac{\Gamma_{2}}{2}\right)^{2}\right]}
\end{aligned}
$$

To fit the experimental data, this function has to be convoluted with a Gaussian representing the energy resolution of the instrument.

The resonant scattering process at the $\mathrm{L}_{\mathrm{II}}$ and $\mathrm{L}_{\mathrm{III}}$ edges in rare-earths involves dipole transitions from the $2 p$ level to the weakly polarized $s$ - and $d$-bands, where the transition to the $5 d$ band is much stronger than that to the $s$-band. $4 f$-levels, can only be probed directly by quadrupolar transitions from the $2 p$ core levels. The dipolar transitions are $2 p_{1 / 2} \rightarrow 5 d$ for the $\mathrm{L}_{\mathrm{II}}$ and $2 p_{3 / 2} \rightarrow 5 d$ for the $\mathrm{L}_{\mathrm{III}}$ edge. The dipolar transition is usually expected to occur at an energy slightly above the edge (inflection point of the resonance) whereas the quadrupolar transition should occur below the edge because of a lowering of the $4 \mathrm{f}$ energy compared with other levels due to the reduced screening of the nuclear charges.

\section{Results}

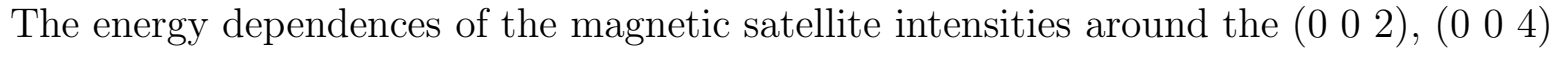
and $\left(\begin{array}{lll}0 & 0 & 6\end{array}\right)$ reciprocal lattice positions have been measured at the $\mathrm{L}_{\mathrm{II}}$ edge as well as at the $\mathrm{L}_{\mathrm{III}}$ edge at a temperature of $\mathrm{T}=224 \mathrm{~K}$ where maximum intensity is obtained. The results for the $\mathrm{L}_{\mathrm{II}}$ edge are shown in figure 3a for the $\left(\begin{array}{lll}0 & 0 & 2\end{array}+\tau\right),\left(\begin{array}{lll}0 & 0 & 4\end{array}+\tau\right)$, and $\left(\begin{array}{lll}0 & 0 & 6\end{array}-\tau\right)$ satellite reflections in $\sigma-\pi^{\prime}$ scattering geometry. The data are corrected for absorption, as described in Section 2. There are two distinct peaks of similar intensities observed, one just below the white line but above the absorption edge and one just above. In addition, a small peak far above the white line at $8265 \mathrm{eV}$ is present which

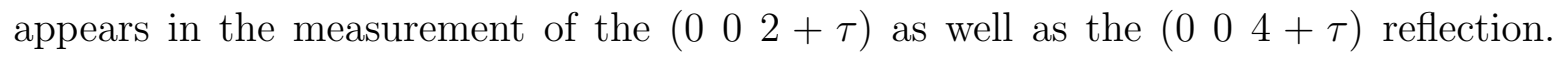


The intensities are normalized to the strong resonance peak above the white line. To show the relative position of the magnetic peaks with respect to the absorption edge, the absorption coefficient as a function of energy is plotted in figure $3 \mathrm{~b}$.

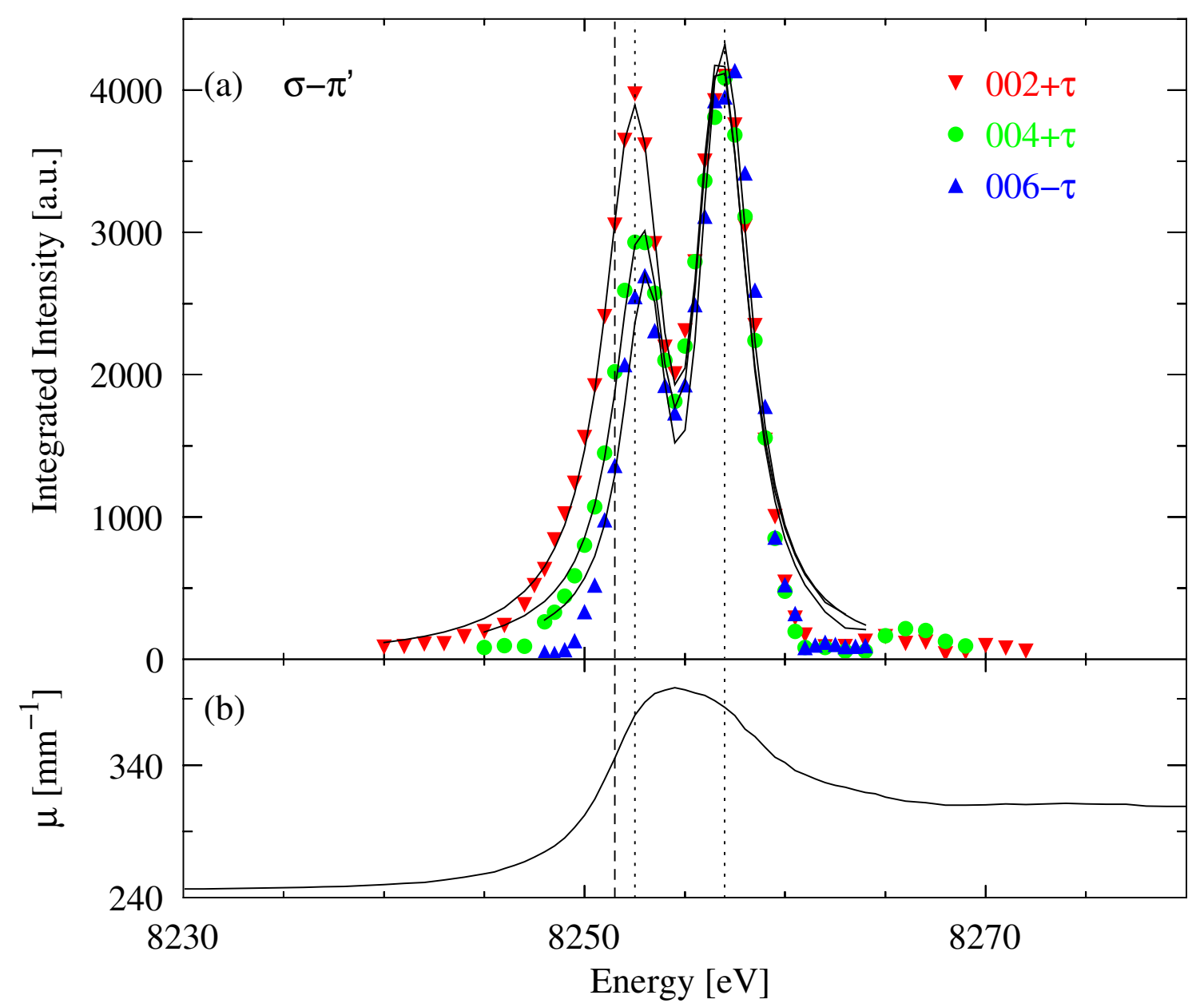

Figure 3. a) Energy dependence at the terbium $\mathrm{L}_{\mathrm{II}}$ edge of the magnetic $\left(\begin{array}{lll}0 & 0 & 2+\tau\end{array}\right)$, $\left(\begin{array}{lll}0 & 0 & 4\end{array}+\tau\right)$, and $\left(\begin{array}{lll}0 & 0 & 6\end{array}-\tau\right)$ satellite reflections. At the bottom (b) the absorption coefficient over the edge is shown. The solid lines represent the fits of Eq. (4) to the experimental data. The vertical dashed line gives the position of the absorption edge. The vertical dotted lines are separated by $4.5 \mathrm{eV}$ and show the position of the resonance peaks.

In figure $4 a$ the energy dependences of the magnetic intensity at the $\mathrm{L}_{\mathrm{III}}$ edge are shown for four different satellites measured in $\sigma-\pi^{\prime}$ scattering geometry: $\left(\begin{array}{l}0 \\ 0\end{array} 2+\tau\right)$,

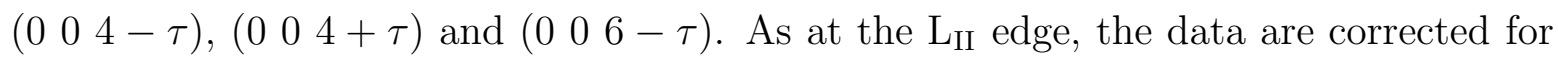
absorption. Here, instead of two distinct peaks as at the $\mathrm{L}_{\mathrm{II}}$ edge only the peak directly at the absorption edge $(7481.9 \mathrm{eV})$, below the white line, is dominant and the peak at higher energies is very weak and is visible only as an extended tail at the respective location above the white line. In addition, a weak peak at lower energies is coming up with increasing momentum transfer.

At the $\mathrm{L}_{\text {III }}$ edge, in addition to the $\sigma-\pi^{\prime}$ channel, also the $\sigma-\sigma^{\prime}$ channel 


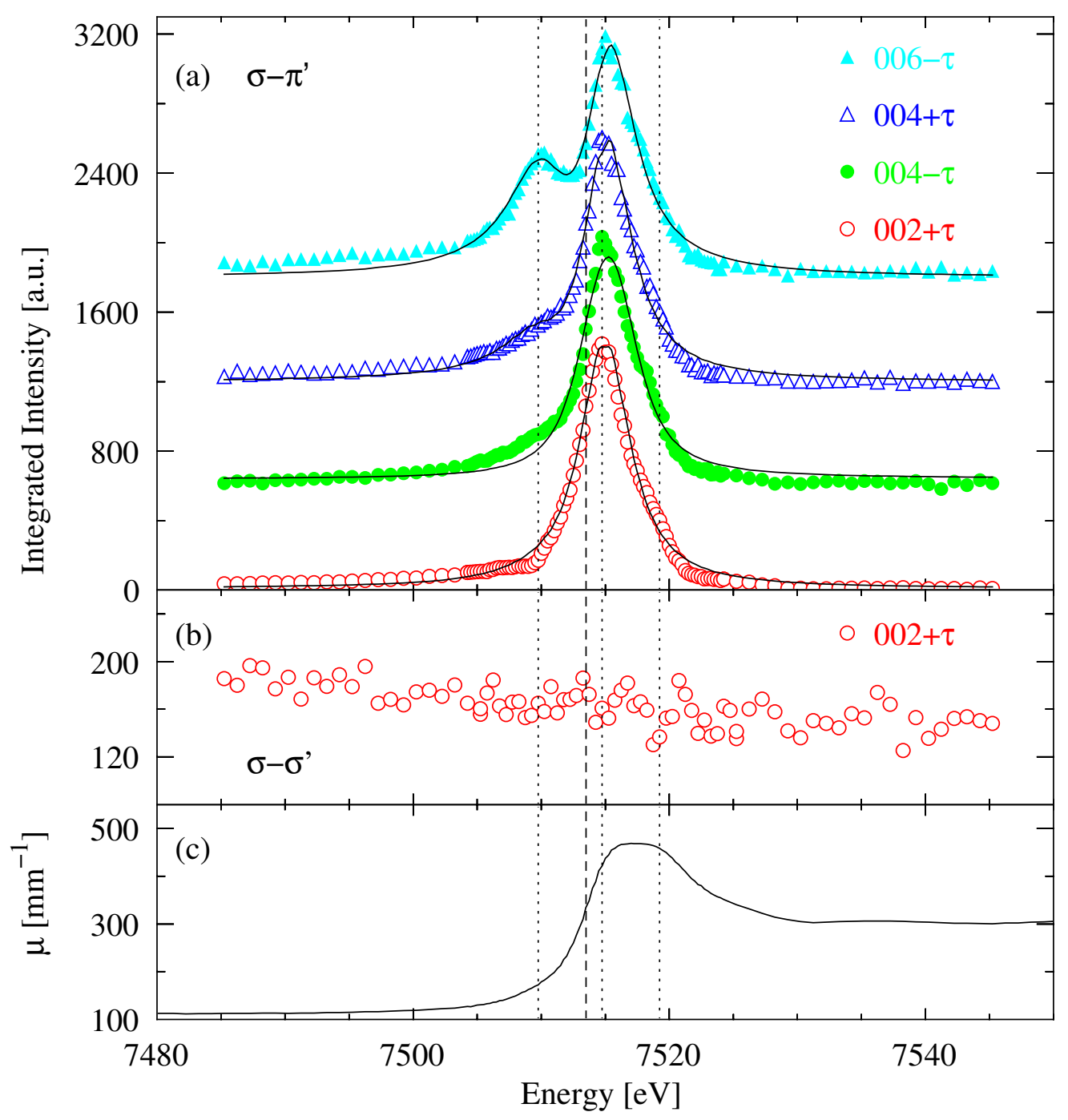

Figure 4. a) Energy dependence at the terbium $\mathrm{L}_{\mathrm{III}}$ edge of the magnetic $\left(\begin{array}{ll}0 & 0 \\ 2 & +\tau\end{array}\right)$,

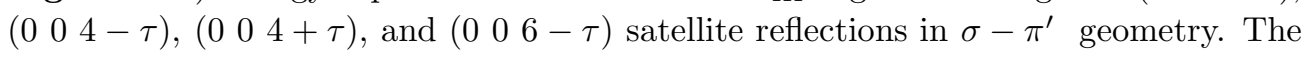
intensities are normalized to be equal in intensity for the higher energy peak. The four curves have an offset of 600 units in intensity to give better visibility. The solid lines represent the fits of Eq. (4) to the experimental data. Below, the result of the $\sigma-\sigma^{\prime}$ polarization analysis at the $(002+\tau)$-position is shown (b). The graph at the bottom (c) shows the absorption coefficient $\mu$ as function of energy. The vertical dashed line gives the position of the absorption edge. The vertical dotted lines give the positions of the resonance peaks. The two peaks at $\mathrm{E}=7510 \mathrm{eV}$ and $\mathrm{E}=7514.9 \mathrm{eV}$ are separated by $4.9 \mathrm{eV}$.

was investigated by performing energy scans at the intensity maximum of the rocking curve. At the $\left(\begin{array}{llll}0 & 0 & 2\end{array}+\tau\right)$ reflection, where according to calculation detailed in [4] a possible quadrupolar contribution should be strongest, no contribution was found. The corresponding $\sigma-\sigma^{\prime}$ signal is shown in figure $4 \mathrm{~b}$. The measurement of the signal in the $\sigma-\sigma^{\prime}$ channel for other positions in reciprocal space was made impossible due to large background signal originating in the main Bragg reflections. 
The peaks at the $\mathrm{L}_{\mathrm{II}}$ and $\mathrm{L}_{\mathrm{III}}$ edge have been fit to a model function using Eq. (4), where two resonant scattering amplitudes for each peak are added. In describing the $5 d$ states we usually are dealing with broad bands of widths of several eV (see figure 7). Nevertheless, as a first approximation an atomic model is assumed in order to be able to perform the fits of the resonant cross section to the energy dependence of the magnetic intensities. The resulting fitting curves are included in figure 3 and 4 . The values obtained for the line-widths, amplitudes and the splitting in energy are given in table 1. No non-resonant contribution to the magnetic signal could be detected. To fit the curves at the $\mathrm{L}_{\mathrm{III}}$ edge, the fit was first performed at the $\left(\begin{array}{lll}0 & 0 & 6\end{array}-\tau\right)$-reflection, where both peaks are clearly visible. For the remaining peaks, the width and position of the lower peak was fixed and only the intensity of this peak and all parameters of the second peak have been varied.

At the $\mathrm{L}_{\mathrm{II}}$ edge, the fits of (4) to the $\left(\begin{array}{lll}0 & 0 & 4\end{array}+\tau\right)$ and $\left(\begin{array}{lll}0 & 0 & 6\end{array}-\tau\right)$-reflections do not agree well with the experimental data in the tails, when the beamline resolution of $\Delta E=1.3 \mathrm{eV}$ is used. Nevertheless, the resolution was kept fixed, since otherwise the line-widths $\Gamma$ would show unreasonably low values.

In figure 5 , the intensity ratios of the two peaks of figure 4 and figure 3, respectively, are displayed. For the $\mathrm{L}_{\mathrm{II}}$ edge, the intensity ratio of the peaks above and below the white line is shown. For the $\mathrm{L}_{\mathrm{III}}$ edge, the intensity ratio between the two peaks below the white line is plotted, where the lower one increases in intensity at high $Q$ with respect to the upper peak. We plot the ratio of the intensities instead of the absolute intensities in order to avoid the influence of geometrical effects due to varying scattering angles.

At the $\mathrm{L}_{\mathrm{II}}$ edge, the energy dependence has been measured for 3 different sample temperatures in the antiferromagnetic phase, $220 \mathrm{~K}, 224 \mathrm{~K}$ and $227 \mathrm{~K}$, at the $(002+\tau)$ reflection. The results did do not show any significant difference in the intensity ratio of the two peaks. The ratio between the peak intensities is 1.01(2). This is supported by the finding that the fluorescence of terbium does not show any change between the antiferromagnetic phase and room temperature.

The fluorescence was measured at the $\mathrm{L}_{\mathrm{II}}$ and $\mathrm{L}_{\mathrm{III}}$ edges and shows some asymmetry as can be seen in figure $6 \mathrm{a}$ for the $\mathrm{L}_{\mathrm{II}}$ edge. The fluorescence of $\mathrm{Tb}$ metal at $224 \mathrm{~K}$ and $\mathrm{RT}$ is compared to the fluorescence of $\mathrm{TbCl}_{3}$. In the latter, the valence state of terbium is purely $\mathrm{Tb}^{3+}$ and consists only of a single line. In comparison, the significant broadening of the white line in $\mathrm{Tb}$ metal becomes obvious. In figure $6 \mathrm{~b}$ the respective second derivatives are shown. The extrema of the second derivative give the positions of the largest change in slope of the fluorescence, showing the location of peak maxima of components within the broad peak. The two extrema observed for $\mathrm{Tb}$ metal coincide with the positions in energy of the magnetic resonances. From the comparison of the observed splitting in energy between the two peaks and the density of states of the 5 d-bands calculated for Tb-metal, a possible conclusion is that the two peaks at the $\mathrm{L}_{\mathrm{II}}$ edge arise from transitions into different energy levels of the 5d-band. 


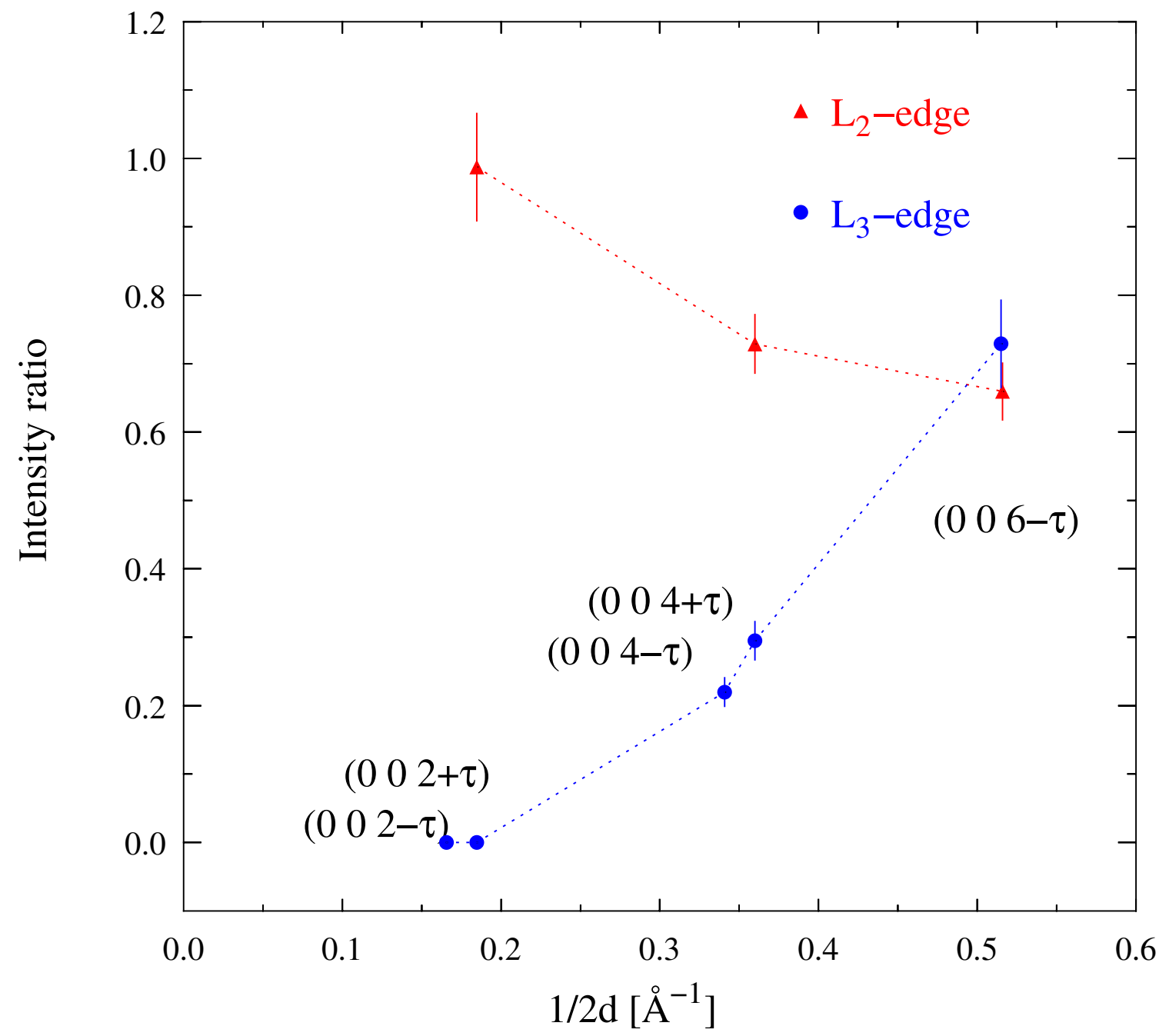

Figure 5. The peak intensity ratios of the energy dependencies shown in figure 3 and figure 4 are displayed. Whereas the ratio for the $\mathrm{L}_{\mathrm{II}}$ edge $(\mathbf{\Delta})$ is taken from the peak below and above the white line, the ratio at the $\mathrm{L}_{\text {III }}$ edge $(\bullet)$ was calculated from the two peaks below the white line in the absorption edge.

\section{Discussion}

According to a calculation presented in [4] dipolar and quadrupolar intensities can be

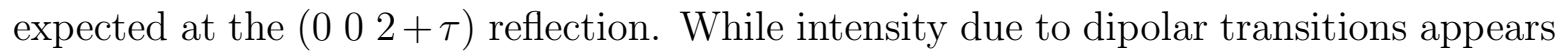
only in the $\sigma-\pi^{\prime}$ channel, intensity due to quadrupolar transitions appears in the $\sigma-\sigma^{\prime}$ as well as in the $\sigma-\pi^{\prime}$ channel. Thus, a possible quadrupolar contribution which is measured in the $\sigma-\pi^{\prime}$ channel should appear purely in the $\sigma-\sigma^{\prime}$ channel without any other contribution superposed. Moreover, for a helical spin arrangement the quadrupolar intensity should vanish completely around $\sin \theta / \lambda=0.45$ as is the case for the $\left(\begin{array}{llll}0 & 0 & 4 & \pm\end{array}\right)$-satellites [4].

From our measurements we can conclude that the intensities measured at the $\mathrm{L}_{\mathrm{II}}$ 


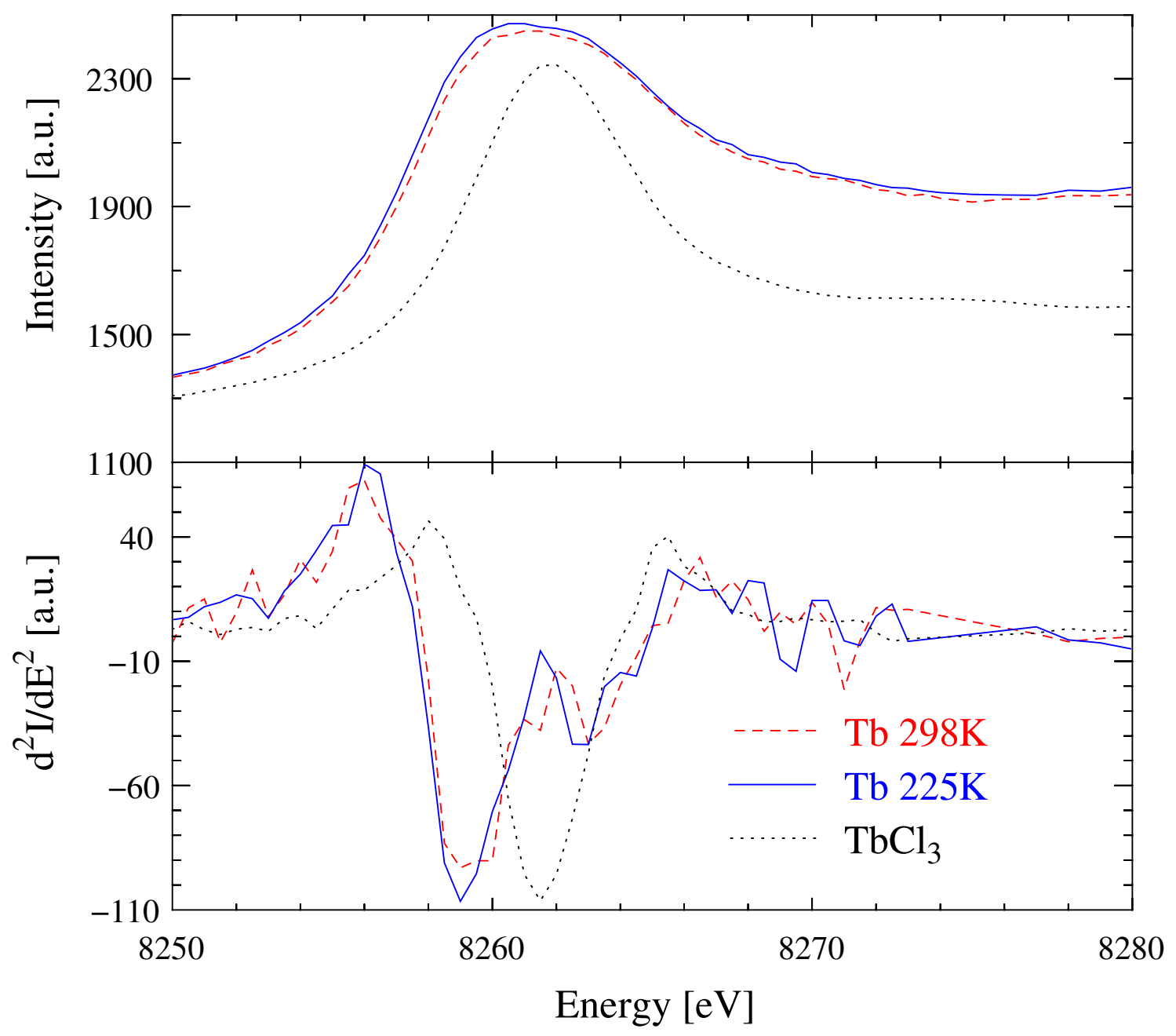

Figure 6. Fluorescence scan over the $\mathrm{L}_{\mathrm{II}}$ edge of pure terbium at a temperature of $298 \mathrm{~K}$ and $225 \mathrm{~K}$. This is compared to the result for $\mathrm{TbCl}_{3}$, measured at room temperature. The second derivative of the intensity with respect to the photon energy is shown as well.

edge are clearly of dipolar nature for both peaks in energy. This conclusion can be drawn from the fact that both peaks appear at the $(004 \pm \tau)$ positions, where the quadrupolar contribution would be zero. Also, the intensity ratio between the two peaks in energy at the $\mathrm{L}_{\mathrm{II}}$ edge measured at this position in the $\sigma-\pi^{\prime}$ channel is the same as the one observed without polarization analysis.

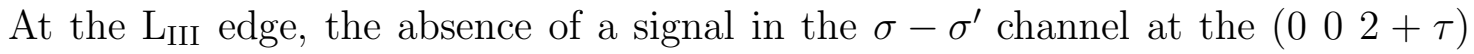
position shows that there is no quadrupolar contribution, as well. According to [4] a possible quadrupolar contribution should be present at this momentum transfer. Therefore, also the small peak below the edge observed at the $\mathrm{L}_{\text {III }}$ edge cannot be of quadrupolar nature, although a possible quadrupolar contribution would be expected right at this position in energy.

Density functional theory calculations employing the full-potential linearized 


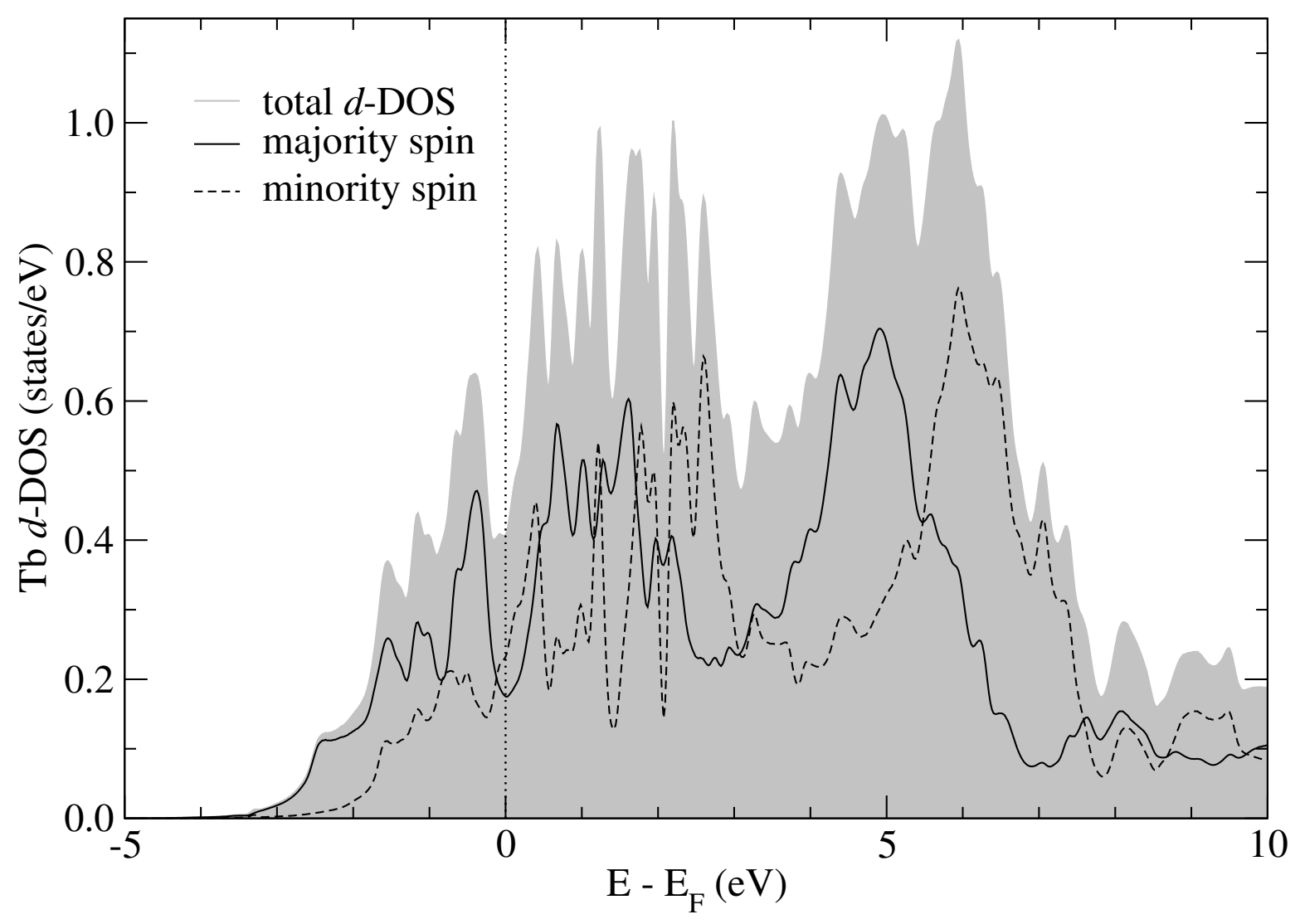

Figure 7. Density of states of the d-like states in Tb metal as function of energy with respect to the Fermi surface. The total density of states (grey shaded area) as well as majority (-) and minority (- - - ) spin density of states are given.

augmented planewave method [21] were performed to evaluate the density of states of the d-like states in Tb-metal, shown in figure 7. For the calculation, a bulk unit-cell with experimental lattice parameters and a spin-spiral with Q-vector of $(0,0,0.1)$ was assumed. Non-collinear magnetism was included in the calculation as described in [22] and the spin-spiral was simulated using the generalized Bloch theorem [23]. All other computational parameters were chosen as given in [24]. A clear splitting of the d-band is found, which corresponds to different crystal field levels. It explains very well the two peaks at the $\mathrm{L}_{\mathrm{II}}$ edge, which are separated by $3.8 \mathrm{eV}$, with electric dipole transitions from the $2 p_{1 / 2}$ levels into the split $5 d$ band. Still not well understood is the difference in Q-dependence of the intensity for the different peaks separated in energy. The variation with $\mathrm{Q}$ of the peak intensity ratio for the different peaks separated in energy, as shown in figure 5, can only be explained by a process involved in the scattering process that is Q dependent as well. Radial matrix elements are not included in this consideration. These are known to be important to explain details in the scattering process and may give an explanation for the observed intensity variations.

For the $\mathrm{L}_{\mathrm{III}}$ edge, the transition probabilities from the $2 p_{3 / 2}$ level into the split $5 \mathrm{~d}$ band seem to be different from the $\mathrm{L}_{\mathrm{II}}$ edge. Whereas the transition into the lower 
part of the band happens with similar probability than at the $\mathrm{L}_{\mathrm{II}}$ edge, the transition probability into the higher part is strongly reduced.

A splitting of the energy dependence of the resonant intensities at the $\mathrm{L}_{\mathrm{III}}$ edge has also been found in the resonance of $\operatorname{Er}(004)$ reflections in [Er|Tb] superlattices [25] showing a peak coming up $5 \mathrm{eV}$ below the edge as has been seen here. In a recent study of rare earth metals in the ferromagnetic phases by x-ray resonant interference scattering at the $\mathrm{L}_{\mathrm{III}}$ edges, a splitting of the $5 \mathrm{~d}$ DOS is indeed found for all heavy rare earth metals [26]. Thus, this peak splitting seems to be a general feature present in the rare earth materials, which has not been observed in the early experiments due to lack of energy resolution.

Table 1. Linewidth obtained by a fit of Eq. 4 to the data convoluted with the fixed instrumental energy resolution of $\Delta E=1.3 \mathrm{eV}$. At the $\mathrm{L}_{\mathrm{II}}$ edge all parameters are refined independently. The peak separation in energy is shown, as well as the ratio of the amplitudes $A_{i}$ and the position in energy. At the $\mathrm{L}_{\mathrm{III}}$ edge only at the $006-\tau$ reflections all parameters are refined.

\begin{tabular}{|c|c|c|c|c|c|c|c|}
\hline Edge & Reflection & $\Gamma_{1}[\mathrm{eV}]$ & $\Gamma_{2}[\mathrm{eV}]$ & $E_{1}$ & $E_{2}$ & $\Delta[\mathrm{eV}]$ & $A_{1} / A_{2}$ \\
\hline $\mathrm{L}_{\mathrm{II}}$ & 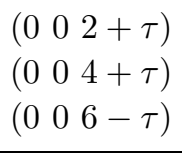 & $\begin{array}{l}2.93(9) \\
2.31(8) \\
1.82(8)\end{array}$ & $\begin{array}{l}2.49(5) \\
2.64(5) \\
2.51(5) \\
\end{array}$ & $\begin{array}{l}8252.8 \\
8253.2 \\
8253.3\end{array}$ & $\begin{array}{l}8256.5 \\
8256.5 \\
8256.6\end{array}$ & $\begin{array}{l}3.8 \\
3.3 \\
3.3 \\
\end{array}$ & $\begin{array}{l}1.152 \\
0.639 \\
0.486\end{array}$ \\
\hline $\mathrm{L}_{\mathrm{III}}$ & 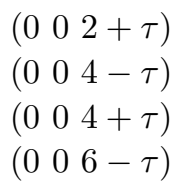 & $\begin{array}{c}- \\
5.04(\text { fix }) \\
5.04(\text { fix }) \\
5.04(4)\end{array}$ & $\begin{array}{c}4.34(7) \\
4.24(9) \\
4.19(9) \\
4.15(12)\end{array}$ & $\begin{array}{c}- \\
\text { fix } \\
\text { fix } \\
7510.0\end{array}$ & $\begin{array}{l}7514.9 \\
7514.9 \\
7514.8 \\
7514.9\end{array}$ & $\begin{array}{c}- \\
4.9 \\
4.8 \\
4.9\end{array}$ & $\begin{array}{c}- \\
0.211 \\
0.281 \\
0.706\end{array}$ \\
\hline
\end{tabular}

\section{Summary}

In this paper we have presented new results on resonant x-ray scattering from terbium obtained with good energy resolution and by using polarization analysis. We could show that the double peak structure in energy is of purely dipolar nature at the $\mathrm{L}_{\mathrm{II}}$ as well as at the $\mathrm{L}_{\mathrm{III}}$ absorption edge. The positions of different peaks are consistent with the density of states of the $5 \mathrm{~d}$-band. The intensity variation over the absorption edges changes when the momentum transfer is changed, which cannot be explained by pure density of state effects. A more detailed theory has to take transition matrix element effects into account. The peak splitting is likely to be a general feature of the $4 \mathrm{f}$ metals, observed here only because of the good energy resolution. Our study demonstrates clearly that an atomic like model is not appropriate to describe XRES in $4 \mathrm{f}$ metals and that solid state effects have to be taken into account for an accurate description of the resonance line shapes. 


\section{Acknowledgments}

We would like to thank K. Attenkofer for fruitful discussions. Work at BESSRC-CAT and use of the Advanced Photon Source was supported by the U.S. Department of Energy, Basic Energy Sciences, Office of Science, Division of Materials Sciences, under Contract No. W-31-109-Eng-38 and the State of Illinois under HECA. The Midwest Universities Collaborative Access Team (MUCAT) sector at the APS is supported by the U.S. Department of Energy, Basic Energy Sciences, Office of Science, through the Ames Laboratory under Contract No. W-7405-Eng-82. This work has been supported

by the BMBF (German Federal Ministry for Education and Research) under contract 05 NA8CJA 8.

\section{References}

[1] Gibbs D, Harshman D R, Isaacs E D, McWhan D B, Mills D and Vettier C 1998 Phys. Rev. Lett. 611241

[2] Gibbs D, Grübel G, Harshman D R, Isaacs E D, McWhan D B, Mills D and Vettier C 1991 Phys. Rev. B 435663

[3] Hill J P, Sternlieb B J, Gibbs D, Detlefs C, Goldmann A I, Stassis C, Canfield P C and Cho B K 1996 Phys. Rev. B 533487

[4] Perry S C, Costa M M R, Sterling W G, Longfield M J, Mannix D and Brückel T 1998 J. Phys.: Condens. Matter 101951

[5] Detlefs C, Goldman A I, Stassis C, Canfield P C, Cho B K, Hill J P and Gibbs D 1996 Phys. Rev. B 536355

[6] Detlefs C, Islam A H M Z, Goldman A I, Stassis C, Canfield P C, Hill J P and Gibbs D 1997 Phys. Rev. B 55 R680

[7] Hill J P, Boothroyd A T, Andersen N H, Brecht E and Wolf T 1998 Phys. Rev. B 5811211

[8] Detlefs C, Abernathy D L, Grübel G and Canfield P C 1999 Europhys. Lett. 47352

[9] Islam Z, Detlefs C, Goldman A I, Bud'ko S L, Canfield P C and Zheludev A 1998 Solid State Commun. 108371

[10] Islam Z, Detlefs C, Goldman A I, Bud'ko S L, Canfield P C, Hill J P, Gibbs D, Vogt T and Zehludev A 1998 Phys. Rev B 588522

[11] Hannon J P, Trammel G T, Blume M and Gibbs D 1988 Phys. Rev. Lett. 611245

[12] van Veenendaal M, Goedkoop J B and Thole B T 1997 Phys. Rev. Lett. 781162

[13] Brückel T, Hupfeld D, Strempfer J, Caliebe W A, Mattenberger K, Stunault A, Bernhoeft N and McIntyre G I 2001 European Physical Journal B 19475

[14] Kim J W, Lee Y, Wermeille D, Sieve B, Tan L, Bud'ko S L, Law S, Canfield P C, Harmon B N, Gorldman A I 2005 Phys. Rev. B 72064403

[15] Tang C C, Stirling W G, Jones D L, Wilson C C, Haycock P W, Rollason A J, Thomas A H and Fort D 1992 J. Magn. Magn. Mater. 10386

[16] Gehring P M, Rebelsky L, Gibbs D and Shirane G 1992 Phys. Rev. B 45243

[17] Hanak J J and Daane A H. 1961 Journal of Less-Common Metals 3110

[18] Hill J P and McMorrow D F 1996 Acta Cryst. A 52236

[19] Brennan S and Cowan P L 1992 Rev. Sci. Inst. 63850

[20] du Plessis P de V, van Doorn C F and van Delden D C 1983 Journal of Magnetism and Magnetic Materials 4091

[21] Wimmer E, Krakauer H, Weinert M, and Freeman A J 1981 Phys. Rev. B 24864

[22] Kurz P, Förster F, Nordström L, Bihlmayer G and Blügel S 2004 Phys. Rev. B 69024415 
[23] Herring C 1966 Magnetism ed G Rado and H Suhl (New York: Academic), Sandratskii L 1986 Phys. Status Solidi B 136167

[24] Döbrich K M, Bihlmayer G, Starke K, Prieto J E, Rossnagel K, Koh H, Rotenberg E, Blügel S and Kaindl G 2007 Phys. Rev. B 76035123

[25] Voigt J, Kentzinger E, Rücker U, Wermeille D, Hupfeld D, Schweika W, Schmidt W and Brückel T 2004 Europhys. Lett. 65560

[26] Brown S D, Strange P, Bouchenoire L, Zarychta B, Thompson P B J, Mannix D, Stockton S J, Horne M, Arola E, Ebert H, Szotek Z, Temmermann W M and Fort D 2007 Phys. Rev. Lett. 99 247401 